\title{
Ion Control for Optimized Imaging with a Novel Gaseous Detector
}

\author{
W.R. Knowles*, M. Toth*, B. Thiel**, R. Schroemges* \\ *FEI Company, 5350 NE Dawson Creek Drive, Hillsboro, OR 97124 \\ ** College of Nanoscale Science and Engineering, University at Albany- SUNY, \\ 255 Fuller Road, Albany, NY 12203
}

A novel detector has been developed for imaging of insulating samples at very high magnification. The detector incorporates a dedicated electrode for the control of the ion flow to ensure correct charge control with any type of sample.

It is well known that the gas ions in the gaseous detector are used to stabilize electron charging in environmental and variable pressure SEMs during the imaging of insulating samples with higher electron beam voltages. However, the gaseous detector can generate many more ions than are needed for charge control. The excess ions do not cause the same artifacts as electron charging in high vacuum but the excess ions cause the surface of the insulator to acquire a positive voltage which reduces image contrast and causes errors in X-ray analysis [1].

Users of the first commercial SEM to incorporate this type of detector (ElectroScan ESEM ${ }^{\mathrm{TM}}$ ) quickly learned that it was easy to image small insulating samples on a metal stub. However, imaging of large insulators was much more difficult unless a conducting path was placed close the imaging area. Copper tape was common solution. The use of ground wires above the sample surface has also been shown to be useful [2]. The ESEM used a high voltage anode directly above, and close to the sample.

A novel detector has been developed to work with magnetic immersion lens for ultra high resolution imaging of insulators [3]. The basic detector is shown in Fig 1. An aperture inside the lens restricts the flow of chamber gas so that the electron column is in high vacuum. The signal collection anode is placed directly between the sample and the lens. Secondary electrons are accelerated by the bias on the anode and follow a complex helical path in the fields from the lens and the anode [3]. The long electron trajectories generate very high gain [4] even though the sample is close to the lens for high resolution imaging. The high gain also creates a large number of excess ions. Imaging of samples larger than $1-2 \mathrm{~mm}$ is difficult with the basic structure of Fig 1.

An enhanced detector is shown in Fig 2. Another electrode, the ion trap, is added between the sample and the anode. Since the ion energy is low (due to collisions with neutral gas molecules) only a small change in sample voltage is required to divert excess ions to the ion trap. The ion trap is normally at ground potential. The Helix detector is very compact, even with the ion trap, allowing imaging at less than $3 \mathrm{~mm}$ working distance.

A simple sample was used to characterize the imaging characteristics of the two detector configurations. This sample consisted of thin PTFE (Teflon) tape covering half of a metal stub. The tape was imaged at various positions relative to the exposed metal stub. Fig 3 shows the variation of image intensity as a function of position (with constant detector settings for each curve). The overall gain for each configuration is different so the data have been normalized. The curves are plotted 
with log scale to show clearly that the image intensity of the basic detector declines continuously with increasing distance. The signal from the Helix detector drops by less than $50 \%$ in the first 400 microns (due to sample changes) and is then stable for larger distances. The detector has been used successfully to image $150 \mathrm{~mm}$ square quartz photomasks [6].

Images showing the charge control of the Helix detector are reported elsewhere in these proceedings [5]. This charge control is applicable to any size insulating sample, due to the use of the ion trap to control the uniformity of the surface potential.

\section{References}

[1] R. A. Carlton, et a.1, Microsc. Microanal., Vol. 10, 753 (2004)

[2] J.P. Craven, et al., J. Microsc. - Oxf., 205 (2002) 96.

[3] B.L.Thiel, et al., Microscopy and Microanalysis (2004) 1058

[4] W.R. Knowles, et al., elsewhere in these proceedings

[5] M. Toth, et al., elsewhere in these proceedings

[6] M.T Postek, et al., Conference on Metrology, Inspection and Process Control for Microlithography XVII, PTS 1 and 2, (2003) 31

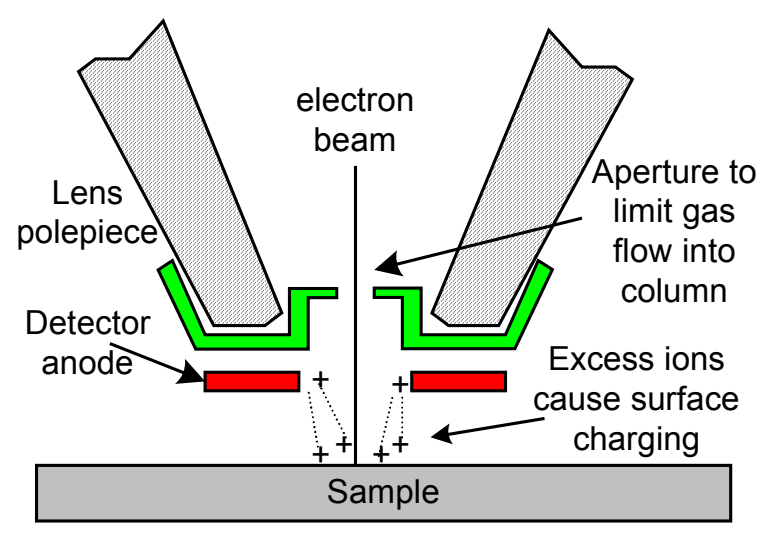

Figure 1 Basic detector

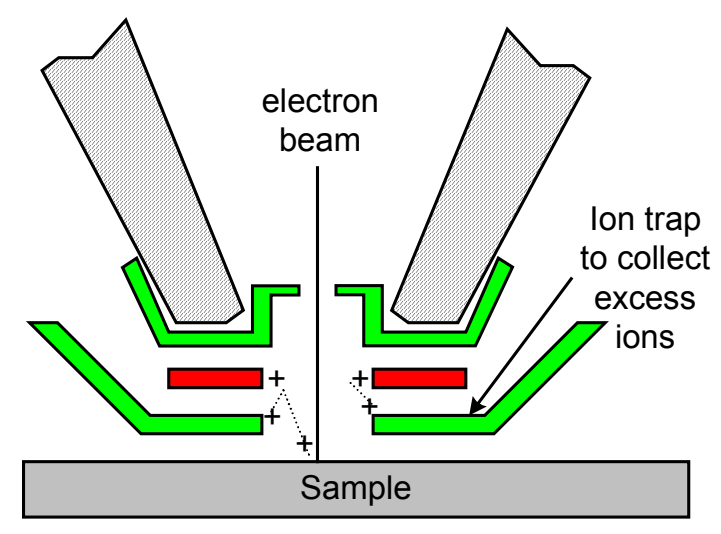

Figure 2 Helix detector with ion trap

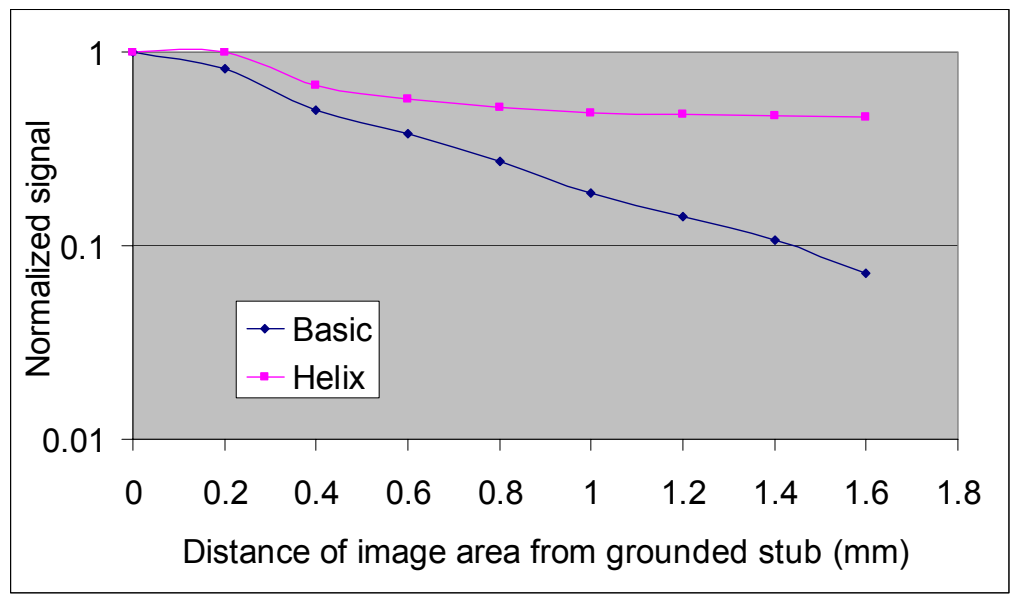

Figure 3 Variation of signal intensity on PTFE with distance from grounded stub 